\title{
DETECTION AND CLASSIFICATION OF CHANGES IN BUILDINGS FROM AIRBORNE LASER SCANNING DATA
}

\author{
S. Xu, G. Vosselman, S. Oude Elberink \\ Dept. of Earth Observation Science, ITC, University of Twente, Enschede, The Netherlands - \\ s.xu, george.vosselman, s.j.oudeelberink@utwente.nl
}

Commission III, III/2

KEY WORDS: Building, change, classification, ALS, change detection, airborne laser scanning

\begin{abstract}
:
Building change detection serves to investigate illegal buildings. Illegal built or removed structures, especially those concealed among gable roofs such as dormers, are difficult to track among potentially millions of buildings. Nevertheless, they can be efficiently located in changed areas. An approach is proposed to automatically detect and classify changes in buildings from two epochs of Airborne Laser Scanning Data. Both datasets are classified into water, ground, building, vegetation and undefined objects in advance. After generalization of a 3D surface separation map, we verify changes by making rules on the separation map. Changes belonging to buildings are then classified into roof, wall, dormers, vehicles, construction above roof and undefined objects. As the ALS data has accuracy in strip difference of lower than $5 \mathrm{~cm}$ within the same epoch and from different epochs, changes that are larger than $10 \mathrm{~cm}$ were detected. Building changes, which areas are larger than $4 \mathrm{~m}^{2}$, are identified as change. By inspection, nearly all changes are detected and approximately $80 \%$ changes are correctly classified.
\end{abstract}

\section{INTRODUCTION}

Illegal building detection is one of the applications of building change detection, besides map updating, disaster and deformation evaluation. Methods for change detection, either using maps or DSMs, pose the problem of information loss. When the change happens under other objects such as vegetation, neither maps nor DSMs can track. Recently, Hebel et al. (2011) track changes by directly compare two epochs of ALS data using occupancy grid. Explicitly comparison using occupancy grid can avoid information loss; furthermore, it can recognize occlusion in 3D environment. Occluded points caused by scanning direction in one epoch usually lead to false change detection when the points in the same location are scanned and seen in the later flight.

Besides occlusion, there also exist other problems: (1) false detection occur when points are lack in one epoch because of water absorption or reflection after rainy days but points in the same location can be visualized in another epoch in sunny days; (2) newly built dormers are detected but change on the roof which is right below the dormer is occluded, and it is a real change. (Illustrated in Figure 1)

This research attempts to detect changed in building by (1) comparing multi-temporal ALS data explicitly by merging them and calculating a 3D surface separation map; (2) making rules over the separation map to solve the occlusion problem as well as the problem we raised above.

The input ALS data are obtained with an accuracy of strip difference below $5 \mathrm{~cm}$, and are in advance classified into ground, water, vegetation, building and undefined objects using Xu et al. (2012)'s method. Changes are identified apart from unchanged and unknown (lack of data in one epoch). Points, which are labelled as changed and are belonging to buildings, are interpreted and classified into changes in roof, walls, dormers, vehicles, constructions above roof and undefined changes.
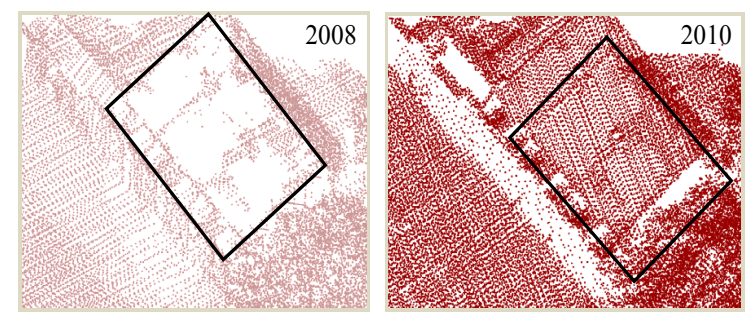

(1) Some points on the roof in 2008 are missed because of water on the roof. But in 2010, the points on the roof are seen. Occupancy grid will recognize this as a change of roof; however, we can observe that there is no change in the building.

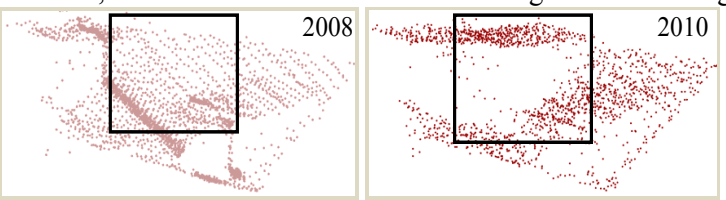

(2) There is a newly built dormer in the rectangle in 2010 . Changes happened on dormer and roof. Normally, changes happen on dormers are easily detected, but changes on roof beneath the dormer are often ignored.

Figure 1 Problems in tracking changes directly in ALS data

Our methodology has three main contributions: (1) changes in building roof, wall and roof element, which differentiate than $10 \mathrm{~cm}$ and area larger than $4 \mathrm{~m}^{2}$, are detected; (2) fake changes such as changed resulted by lack of data are identified as unknown; (3) changes are interpreted by classification. After literature review in Section 2 and data introduction in Section 3, 
we introduce the change detection method in 4.1 and 4.2 . Method for classification of changes is explained in 4.3. The results are shown and discussed in Section 5. Conclusions are drawn in Section6.

\section{RELATED WORKS}

There are two basic approaches to the problem of change detection: (i) Original data is available to both epochs, in which change detection is used for disaster or deformation evaluation. (ii) Original data is only available to one epoch, and the other epoch is an existing map or database. Change detection in this case is used for map updating. (Vosselman et al., 2004)

Approach 1. In 1999, Murakami et al. (1999) used two ALS datasets in 1998 and in 1996 to detect the changes of building in the dense urban area in Japan after an earthquake. A difference map was obtained by subtracting one DSM from another. The difference map was overlaid on an ortho-image and existing GIS database to recognize changes belonging to buildings. Vögtle and Steinle (2004) presented their work as a part of a project aiming at detecting changes after strong earthquakes using DSMs from two ALS datasets. Segmentation was run to get separate 3D objects. Changes are identified by the overlay rate of all the buildings in both epochs. Rutzinger et al. (2010) picked out foot print by using classification tree after DSMs from two epochs were segmented. The shape index and mean height difference of the segments were compared. Choi and Kim (2009) also used the difference DSMs to detect all changes and finally gave the clues of changes categories in two epochs' ALS data.

Approach 2. Vosselman et al.(2004) used ALS data to compare ALS data with an existing medium scale map. The change detection was executed by segmentation, classification and the map rule implementation. Pixel overlay rates on classified DSM and a raster map formed the final judgement for changes. This method was then improved by using aerial image to refine the classification results (Matikainen et al., 2004). Rottensteiner (2007) employed Demster-shafer data fusion theory for building detection. He then improves his method by adding one more feature into the data fusion, which makes the classification suitable for building change detection using a map and a DSM. Champion et al. (2009) detected building changes by generating DSM from ortho photos and then compared DSM with a vector map. The similarity measure between the vector map building outlines and the DSM contours were used to identify the destroyed, modified and validated, and new buildings were separately detected in the DSM. Chen, et al. (2010) used the Lidar data and the aerial image to update the old building model. After registration of the data, change area was detected and height differences were calculated between the roof planes in Lidar data and the old model planes. A double-thresholding strategy was used to identify main-structure changed area and unchanged area, uncertain parts were identified by the line comparisons between building boundaries extracted from aerial image and the projection of old building models. Doublethresholding method was reported to improve overall accuracy from $93.1 \%$ to $95.9 \%$.

Changes are all detected in 2D (maps) or 2.5D (DSMs) in previous works. In 2011, Hebel et al. (2011) employed occupancy grid to track changes explicitly in multi-temporal 3D ALS data. In order to know how a laser beam affects the other, they defined belief masses \{empty, occupied, unknown\} for the effected laser beam, making use of the longitudinal and transverse distance between two points. For the compared datasets, they computed the belief masses for all the laser beams, and conflicts in belief masses denote a change. With this method, they achieved reliable change detection results even when occlusion occurs in either of the epoch. However, in case of an illegal dormer or false change resulted by rains as mentioned in Section 1, occupancy grid fails.

\section{DATASET}

The Municipality of Rotterdam is responsible for the building enforcement and the property management of Rotterdam. To verify the building permits, they need to track changes in buildings in metropolitan environment with potentially million of construction. They cooperated with the Fugro Company and obtained multiple temporal ALS data of Rotterdam in 2008, 2010 and 2012. We were offered the opportunity and the data to automatically track changes in building.

Two test areas are located in commercial and residential places in Rotterdam, the Netherlands. Commercial group was scanned in year 2008 and 2010, and residential group was in year 2010 and 2012. The point densities for dataset 2008, 2010 and 2012 are $20-30 \mathrm{pts} / \mathrm{m}^{2}, 30-40 \mathrm{pts} / \mathrm{m}^{2}$ and $40-50 \mathrm{pts} / \mathrm{m}^{2}$. The strip difference within one epoch and between different epochs is below $5 \mathrm{~cm}$, and therefore all data are well registered. All datasets were classified with the method in (Xu et al., 2012). Buildings are detailed as roof element, roof and wall. One classified example can be found in Figure 10.

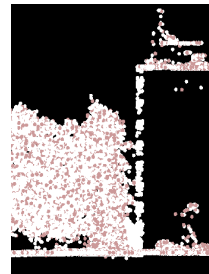

(a)

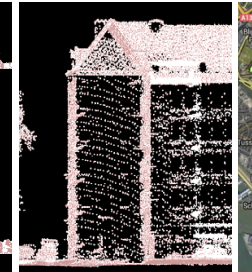

(b)

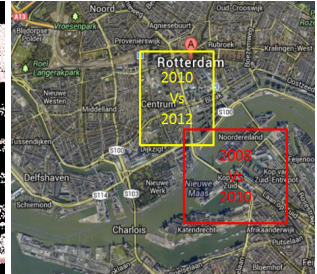

(c)
Figure 2 (a) Merged data from different strip within the same epoch; (b) different strip in different epoch; Different colours represent different strip. (c) Test Area.

\section{METHOD}

A three-dimensional-map is generated point wise by calculating surface separation in a merged datasets, which was described in Section 4.1. Making use of the surface separation values, we distinguish the changed from unchanged and unknown with a series of rules as introduced in Section 4.2. Points, which are identified as changed on buildings, are finally classified into: roofs, walls, dormers, vehicles, construction and undefined changes, by a knowledge-based classifier as explained in Section 4.3.

\subsection{Three-Dimension Surface Separation Map}

3D Surface Separation Map (SSM) records the disparities of points between two epochs of ALS data. The disparity was computed as "the distance from a point to its nearest fitted plane from a distinct epoch". Surface separation was once employed by (Vosselman, 2012) to evaluate the quality of data among overlap strips. It is used to indicate a possible change.

To calculate SSM, two datasets were merged as one with distinguishing epoch numbers. For every point in the merged 
dataset, we searched within its $1.0 \mathrm{~m}$ in $3 \mathrm{D}$ to check whether there is a point from another epoch. " $1.0 \mathrm{~m}$ " was chosen because most large changes are larger than one meter. Absence of point from different epoch denotes a difference larger than $1 \mathrm{~m}$ which was recorded with 4 or -4 in the SSM. Presence of points from another epoch indicates a difference smaller than $1.0 \mathrm{~m}$ or no difference, and was recorded as "the distance from the point to its nearest fitted plane from another epoch" in SSM. Equation 1 illustrated the formulation of the SSM, and the process is charted in Figure3. One example of SSM is shown in Figure 4.

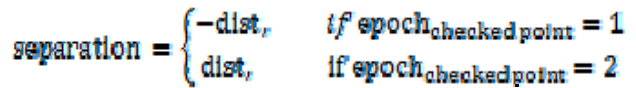

dist - distance to the nearest plane from another epoch dist $=4$; if no point from another epoch is found within $1.0 \mathrm{~m}$.

Equation 1 Calculation of SSM

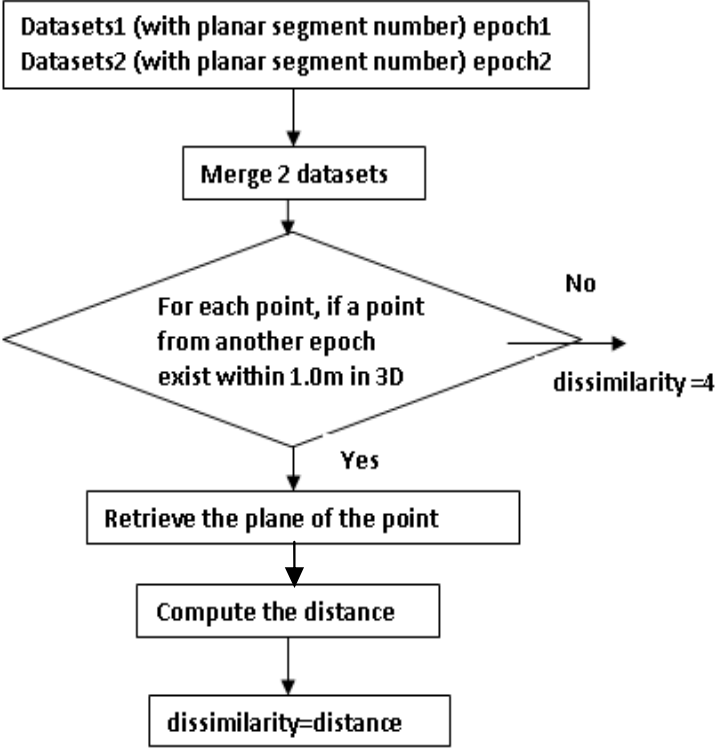

Figure 3 Flow of forming the SSM

\subsection{Identification of Changed Building Points}

During SSM generation, we calculated the distance from the point to it nearest plane. These planes were fitted in such a way: points, which have a distance smaller than $10 \mathrm{~cm}$ to the plane, were added to the plane for calculation of the plane. Therefore, we can only detect changes that are larger than $10 \mathrm{~cm}$, and it will not be effected by the strip difference as the difference is below $5 \mathrm{~cm}$.

We cannot assure a change by simply thresholding the SSM. Points, which have the distance separation larger than $10 \mathrm{~cm}$, are not always changed (Figure 5). Reversely, points with separation smaller than $10 \mathrm{~cm}$ maybe changed (such as showed in Figure 6). Besides changed and unchanged, there are some unknown areas. For instance, presence of points in one epoch and absence of point resulted by data absorption or occlusion in another epoch may yield changes as well, and it happened frequently on walls and roofs (Figure 5). To differentiate among \{changed, unchanged, unknown\}, rules are defined separately.

Unknown points: Among the points with separation value larger than $1 \mathrm{~m}$ in SSM in the merged dataset. An "unknown" is affirmed if no point from distinct epoch was found both in 3D and $2 \mathrm{D}$ neighbourhood. In Figure 7, there is no data on the building wall in 2008. However, there are points on wall in 2010. The changes on wall are unknown.

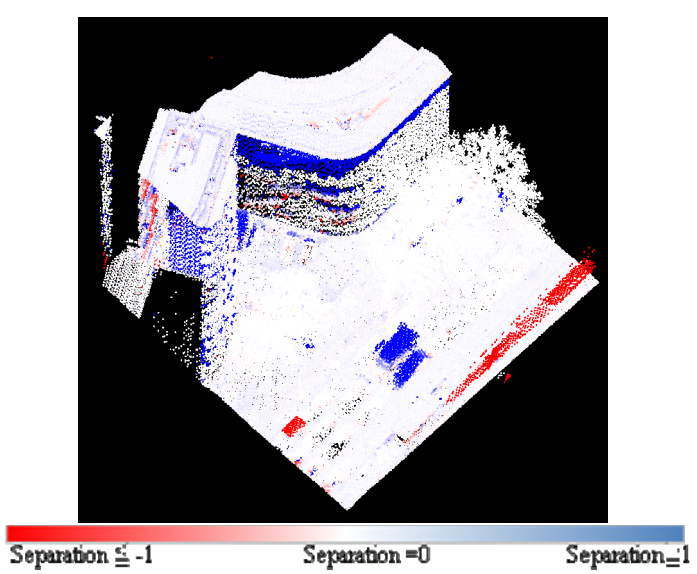

Figure 4 One result of a SSM, red points means possible demolished points, and blue represents possible new points.

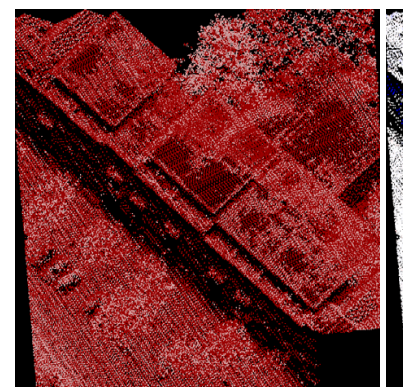

(a)

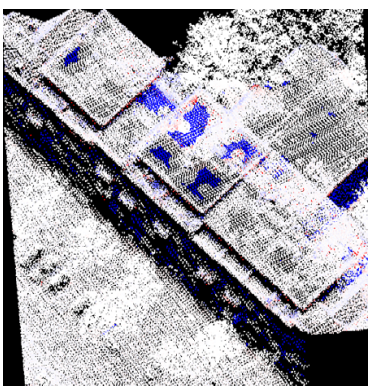

(b)

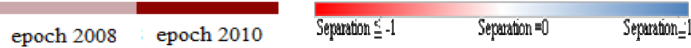

Figure 5 (a) merged dataset. Different colour represents different epoch. (b) SSM. Points with a high separation do not always denote a change, for example, blue points in (b) are unknown points because there is no data in epoch 2008, although they have high separation values.

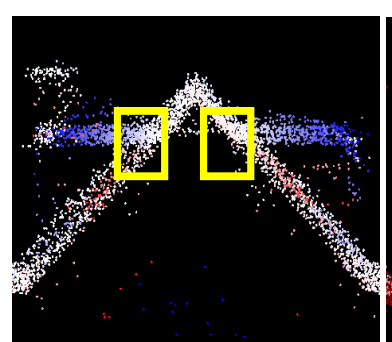

(a)

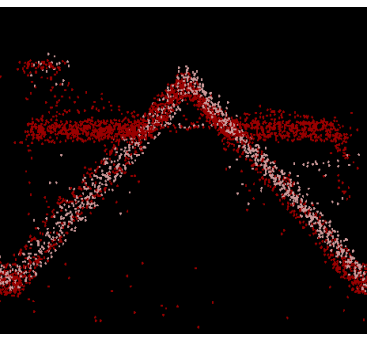

(b)
Figure 6 (a) Merged datasets (b) SSM. The points in the yellow rectangle have a distance separation of $8 \mathrm{~cm}$ or even smaller, however, they are indeed changed. The challenge is to identify the areas in rectangle as changed. (the colour bar is in Figure 5)

Changed points: Except unknown, left points were grouped as planar segments with the surface growing method (Vosselman et al., 2004). Within each segment, points are separated into compact components in case two objects are close and belong to same plane (an example is shown in Figure 9(a)).

In each compact component, if $80 \%$ of points have the separation larger than $10 \mathrm{~cm}$, all points in the component are 
labelled as changed. Besides this, only points with separation value larger than $1 \mathrm{~m}$ are labelled as changed (Figure 9(b)). A flow chart of the process can be found in Figure 8 .

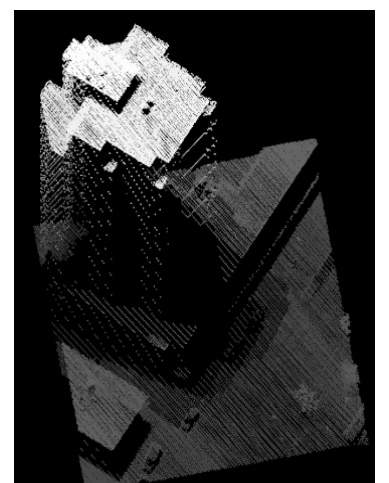

2008

(Lighter grey means larger height)

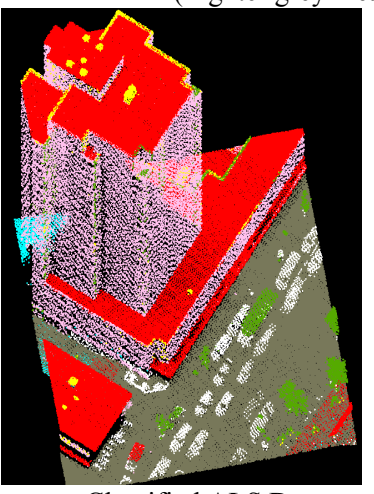

Classified ALS Data

2010
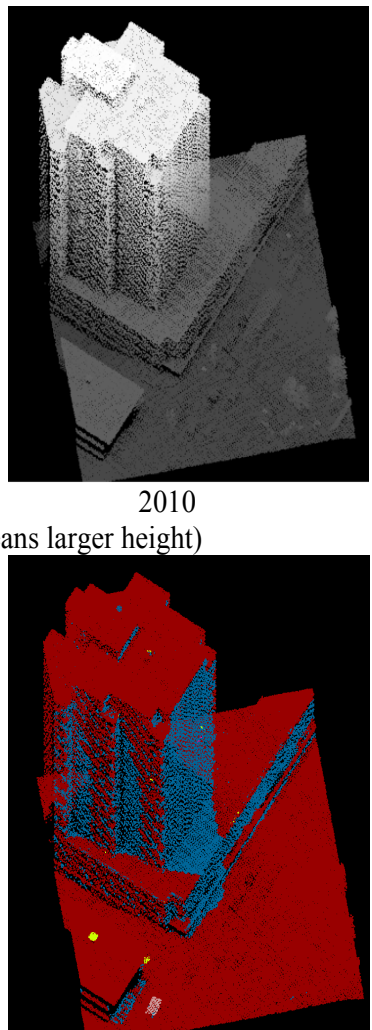

Blue - unknown points

(Shown in Merged datasets)

Figure 7 Unknown points. Data on walls in 2008 is missing as shown in the upper image in 2008, but in 2010 there are points on walls. Lower column image is a classification result on the

left and on the right points in blue are unknown.

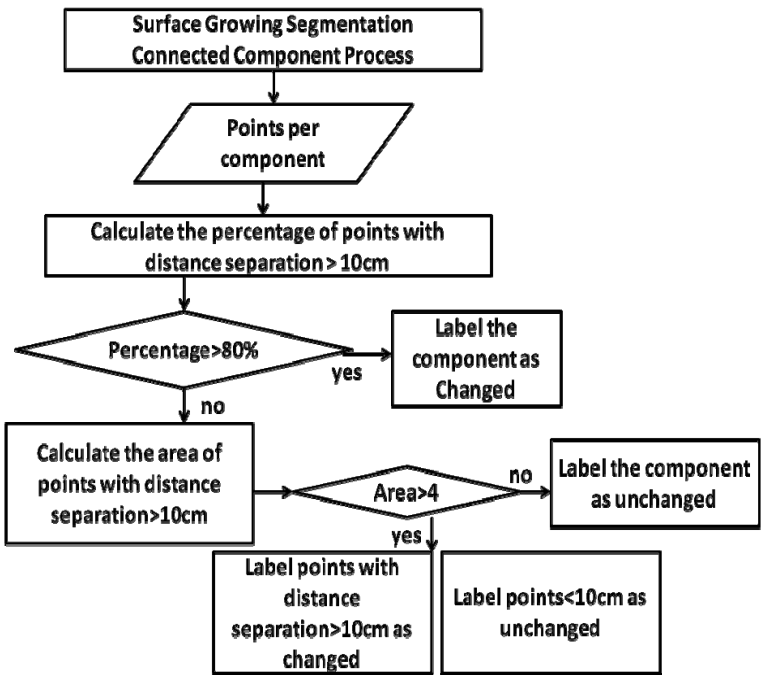

Figure 8 Identification of changed and unchanged points

Unchanged: Except points labelled as unknown and changed, left points are all labelled as unchanged. A result including points assigned as changed and unchanged is shown in Figure 10. In Figure 10 (a), two datasets are merged into one and we can observe new dormers on the gable roofs. In Figure 10 (b), these dormers are detected as changed. Dormers in light green points were there in 2008 but disappeared in 2010. Lavender points are new dormers in 2010 . This indicated heightened and enlarged dormer in 2010 compared with 2008.

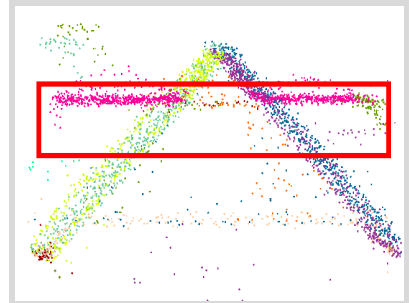

(a)

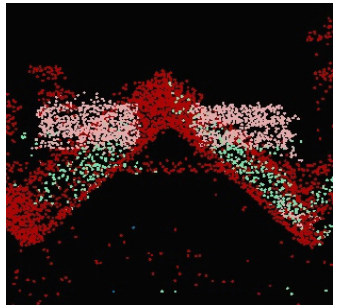

(b)
Figure 9 (a) Two dormers in the red rectangle belong to the same planar segment, and therefore need compact components to separate them. (Different colour indicate different segments); (b) both changes on roof and dormers are detected (two epochs are merged)

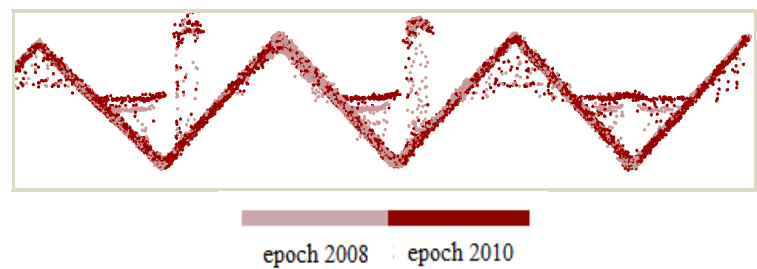

(a) Merged data different colours indicate different epochs

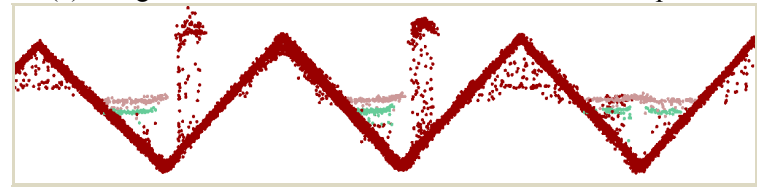

(b) Lavender-New points; light green - demolished points; Blue-Unknown; Red-Unchanged. unchanged demolished new

Figure 10 Changed and unchanged points

\subsection{Classification of Changed Building Points}

Building points, which are labelled as changed, are selected. The changed points on buildings are classified per component into roof, wall, dormer, vehicle, construction above roof and undefined change. The sign of the distance separation value indicates if they are new or demolished. The attributes that used for classification are: area, height to the nearest roof, normal of nearest roof (type of the roof) and class label of point as listed in Table 1.

Table 1. Attributes to classify changed points

\begin{tabular}{|l|l|}
\hline Attribute Name & Abbreviation \\
\hline Area & Area \\
Height to the nearest roof & H_to_nroof \\
Normal of nearest roof(Roof Type) & Tpye_nroof \\
$\begin{array}{l}\text { Class label of point in advance } \\
\text { (roof, roof element, wall) }\end{array}$ & C_of_point \\
\hline
\end{tabular}

Large area of points labelled as changed indicates a roof. Small changes happen on gable roof are normally dormers or chimneys, and if changes occur on a flat roof, they are probably constructions above roof or vehicles. This knowledge are interpreted into rules, and established as a rule-based classifier. (Figure 11) All thresholds used, such as area larger than $4 \mathrm{~m}^{2}$, 
height larger $3 \mathrm{~m}$, are chose in such a way as human recognize objects.

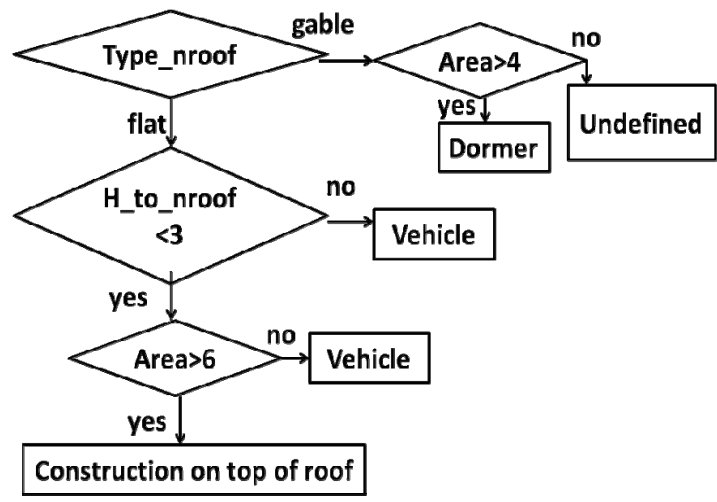

Figure 11 Rule-based classifier for classification of changes

\section{RESULTS}

Results for the two test areas can be visualized in Figure 12 in the end of the paper. Some classified changes are visualized in Figure 13 in merged datasets. As we do not have ground truth data to evaluate the results, we chose 17 sites to manually inspect whether the changes are found and properly classified. Among 9 changed dormers, all of them are detected as changed and 7 dormers are classified as dormer on roof, 2 dormers are wrongly classified as construction above roof. Vehicles are all detected as change, but nearly $1 / 3$ of the vehicles are wrongly classified into construction. Extended building roof, added on building roof, newly built and demolished building roofs are all detected and correctly classified.

These 17 sites include: (a) newly built dormers on roof, (b) lack of data on roof in one epoch (unknown), (c) undefined changes on roof, (d) newly built construction above roof, (e) vehicles parked on top of building, (f) newly built building and demolish building, (g) extended dormers on roof. Through inspection, we found all changes were detected. However, there are failures in classification of these changes as circled in irregular circles.

Changes from vehicles are classified as constructions above roof in Figure 13(e). Because these vehicles are close to other construction on roof, and were taken as one component to process. Dormers, which are extended and heightened, are partly classified as dormer and one of these dormers are classified as construction Figure 13(g). This is mainly caused by detecting a wrong nearby roof such as the dormer itself which is not gable but flat.

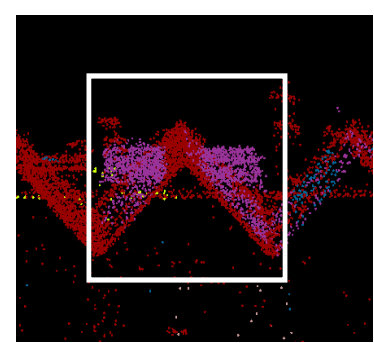

(a)

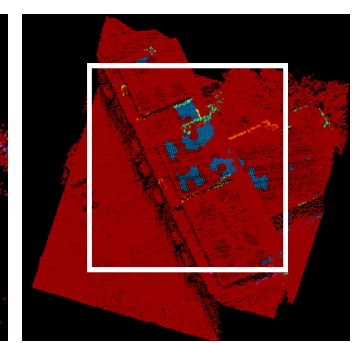

(b)

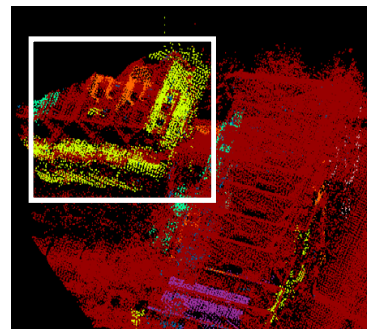

(c)

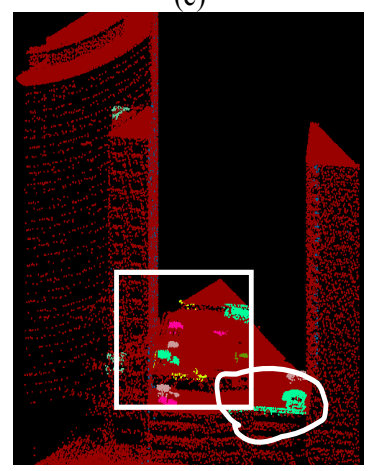

(e)

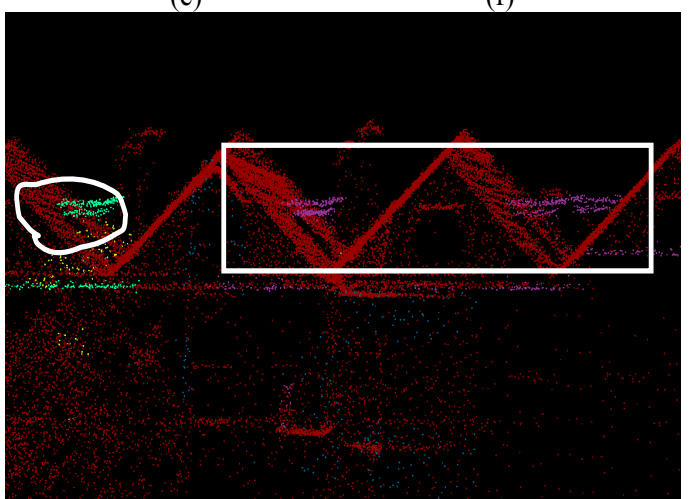

(g)

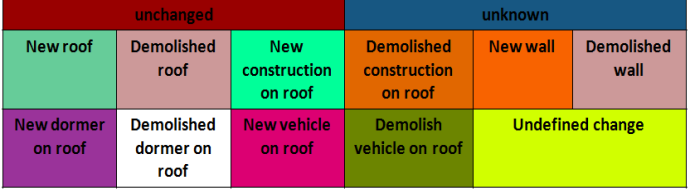

Colour table - change category

Figure 13 Results of detected changes and interpretation.

\section{CONCLUSIONS}

We detected changes in building in multi-temporal ALS data by employing surface separation. The laser data is already registered with a maximum absolute systematic offset of $5 \mathrm{~cm}$. It is our intention not to confuse registration errors with object changes. Changes, which differentiate more than $10 \mathrm{~cm}$ and area larger than $4 \mathrm{~m}^{2}$, are reliably determined by defining rules over the separation values. Approximately, $80 \%$ (estimated by inspection) of building changes are interpreted correctly. Changes of vehicles are wrongly classified when they are near some big construction above roof or when they have an area larger than 4. Changes from extended dormers are easily incorrectly classified as construction above roof because the extended dormer is classified as roof and the nearest roof is the dormer which is flat. The results will be used as assistant data for Municipality of Rotterdam to tax on illegal constructions. 


\section{REFERENCES}

Champion, N., Rottensteiner, F., Matikainen, L., Liang, X., Hyyppä, J., Olsen, B. P., 2009, A test of automatic building change detection approaches, In:Stilla U, Rottensteiner F, Paparoditis N(Eds) CMRT09, The International Archives of Photogrammetry, Remote Sensing and Spatial Information Sciences, Vol. XXXVIII, Part 3/W4, pp.145-150.

Chen, L., Lin, L., Cheng H.,Lee, S., 2010, Change detection of building models from aerial images and Lidar data, The International Archives of Photogrammetry, Remote Sensing and Spatial Information Sciences, Vol. XXXVIII, Part 7B, pp.121126.

Choi, K., Lee, I., Kim, S., A feature based approach to automatic change detection from Lidar data in urban areas, The International Archives of Photogrammetry, Remote Sensing and Spatial Information Sciences, Vol. XXXVIII, Part 3/W8, pp.259-264.

Matikainen, L., Hyyppä, J., Kaartinen, H., 2004, Automatic detection of changes from laser scanner and aerial image data for updating building maps, XXth ISPRS Congress, Istanbul, Turkey.

Murakami, H., Nakagawa, K., Hasegawa, H., Shibata, T., Iwanami, E., 1999, Change detection of buildings using an airborne laser scanner, ISPRS Journal of Photogrammetry and Remote Sensing, Vol. 54 , Issues 2-3, July 1999, pp.148-152.

Rottensteiner, F., 2007, Building change detection from digital surface models and multi-spectral images, The International Archives of Photogrammetry, Remote Sensing and Spatial Information Sciences, Vol. 36, Part 3/W49B, pp.145-150.
Rutzinger, M., Ruf, B., Hofle, B., Vetter, M., 2010, Change detection of building footprints from airborne laser scanning acquired in short time intervals, In: Wagner W., Szekely. .(eds.):ISPRS TC VII Symposium-100 Years ISPRS, Vienna, Austria, July 5-7, 2010, The International Archives of Photogrammetry and Remote Sensing, Vol. XXXVIII, Part 7B, Pp.475-480.

Vosselman, G., Gorte, B.G.H., Sithole, G., Rabani, T., 2004, Recognising structure in laser scanner point clouds. In: The International Archives of Photogrammetry, Remote Sensing and Spatial Information Sciences, Freiburg, Germany, Vol. XXXVI, Part 8/W2, pp. 33-38.

Vosselman, G., 2012, Automated planimetric quality control in high accuracy airborne laser scanning surveys, ISPRS Journal of Photogrammetry and Remote Sensing, Vol. 74, pp.90-100.

Vosselman, G., Gorte, B.G.H., Sithole, G., 2004, Change detection for updating medium scale maps using laser altimetry. The International Archives of the Photogrammetry, Remote Sensing and Spatial Information Sciences, Vol. 34, Part B3, pp.207-212.

Vögtle, T., Steinle E., 2004, Detection and recognition of changes in building geometry derived from multi-temporal laser scanning data, The International Archives of Photogrammetry, Remote Sensing and Spatial Information Sciences, Vol. 34, Part B2, pp.428-433.

Xu, S., Oude Elberink, S., Vosselman, G., Entities and features for classification of airborne laser scanning data in urban area, 2012, ISPRS Annals of the Photogrammetry, Remote Sensing and Spatial Information Science, Vol. I-4, pp.257-262.

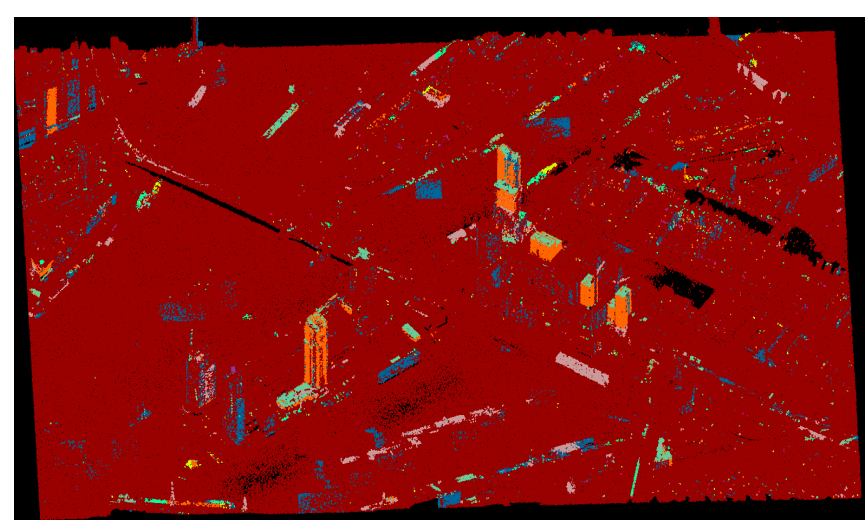

(a) 2008 compared with 2010

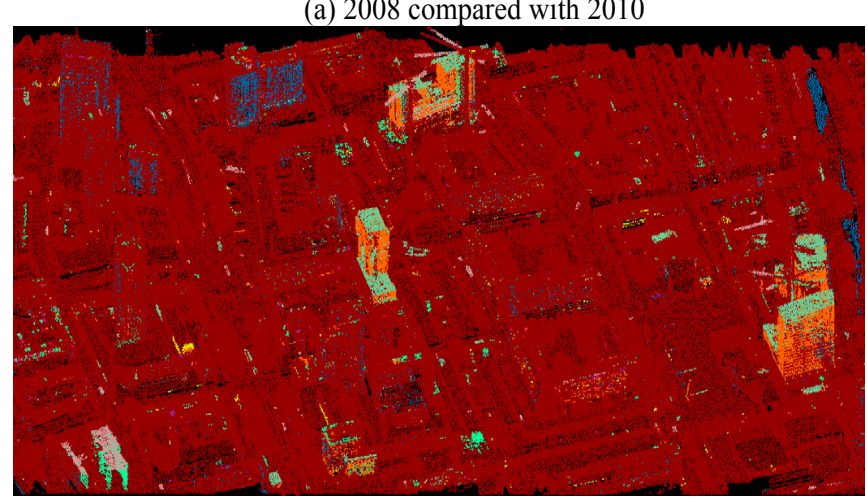

(b) 2010 compared with 2012

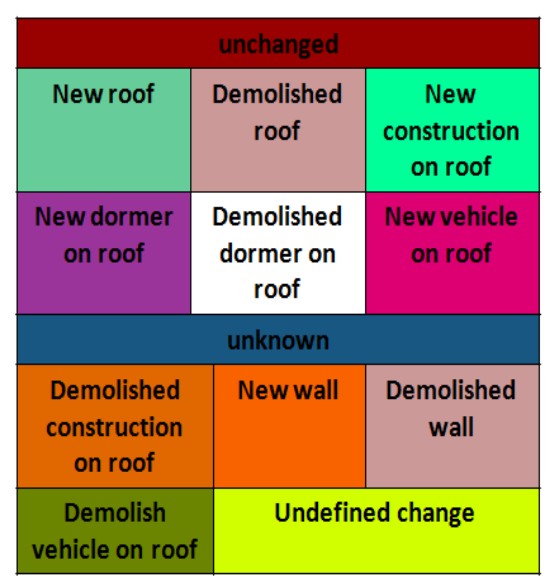

(c) Colours for type of change

Figure 12 Overview of changes for two test areas 